\title{
Original
}

\section{Comparison of Bone Histomorphology and Bone Mineral Density in Different Parts of Ovariectomized Osteoporosis Rats}

\author{
Sainan Chen ${ }^{1)^{*}}$, Yu Lin ${ }^{2 *}$, Shengqiang $\mathrm{Li}^{1)}$, Yunjin $\mathrm{Ye}^{1)}$, Lihua Xie ${ }^{1)}$, Juan Chen ${ }^{1)}$, Huasong $\mathrm{Wu}^{3)}$, Youmin Cheng ${ }^{3)}$ and Jirong $\mathrm{Ge}^{1)}$ \\ 1) Key Research Laboratory of Osteoporosis Syndrome Genomics, Fujian Academy of Traditional Chinese Medicine, Fuzhou, China \\ 2) Orthopedic, the Second Hospital of Fuzhou Affiliated to Xiamen University, Fuzhou, China \\ 3) Center for Comparative Medicine, Fujian Academy of Traditional Chinese Medicine, Fuzhou, China \\ (Accepted for publication, February 28, 2019)
}

\begin{abstract}
This study aimed to investigate the changes of histomorphology and bone mineral density (BMD) change in different parts of ovariectomized osteoporosis rats. The six-month SD rats were randomly divided into the sham operation group and operation group, each consisting of 30 rats and an osteoporosis model was established by bilateral ovariectomy. The rats were harvested at 8 weeks, 12 weeks and 16 weeks after operation. The changes of bone morphology were observed by Masson staining on lumbar spine 3-5, right tibia and femur. BMD was measured at lumbar spine, left tibia and femur by Dual Energy X-ray Absorptiometry (DXA). Compared with sham operation group, the trabecular bones of the model group were sparse, irregularly arranged, unevenly distributed, the trabecular bone spacing was enlarged. A significantly increased amount of lipid droplets in the medullary cavity. The bone mass changes were most significant at 16 weeks after operation, reflected by the significant trabecula loss in the proximal tibia and distal femur, while in distal tibia and lumbar spine trabecula loss is less significant; BMD declines in all the four parts $(P<0.01)$ and the differences in BMD, in descending order, are the proximal tibia, distal femur, proximal femur, and lumbar spine. In ovariectomized rats, the most sensitive areas to bone mass change are the proximal tibia and distal femur, which are major weight-bearing bones; non-weight-bearing areas show the most significant trabecula loss, and there is a positive correlation between the degree of trabecula loss and lipid accumulation in the medullary cavity, which makes non-weight-bearing areas optimal for osteoporosis research on postmenopausal rats.
\end{abstract}

Key words: Postmenopausal osteoporosis, Ovariectomy, Bone mineral density, Masson staining

\section{Introduction}

Caused by a variety of factors, osteoporosis is a systemic metabolic bone disease including reduced bone mass per unit volume, bone microarchitecture degeneration, bone mineral density decline, and increased bone fragility, which leads to a higher risk of fracture ${ }^{1,2}$. PMOP is a more common form of osteoporosis ${ }^{3,4}$. As a result of the rapidly aging population, PMOP gradually increases ${ }^{5)}$. With fracture-induced deformity and disability as the most common serious complications ${ }^{6,7)}$ and involving a huge number of sufferers and expensive medical treatment, it seriously decreases quality of life in older adults and poses a major burden on their families and the society ${ }^{8,9)}$. Therefore, the prevention and treatment of PMOP have been a focus of relevant studies, while postmenopausal osteoporosis animal model serves as a basis for conducting such studies.

As a classic method to create the postmenopausal osteoporosis mod$\mathrm{el}^{10,11)}$, bilateral ovariectomy in rats has been a preferred choice of most researchers. Varying degrees of bone density decline have been found in different areas of ovariectomized rats. However, bones of quadruped-

* Both authers share first place.

Correspondence to: Dr, Jirong Ge, Key Research Laboratory of Osteoporosis Syndrome Genomics, Fujian Academy of Traditional Chinese Medicine, Fuzhou, Fujian, China; Tel: +86-13706957292; Fax: +86-0591-83570943; E-mail: gjrrjgcy@163.com al-walking rodents actually differ in weight bearing from upright walking humans, plus that different areas are observed in different osteoporosis studies ${ }^{12-15)}$, leading to a low probability for replication and comparison.

For this reason, this study built a postmenopausal osteoporosis model through ovariectomizing rats and collected bones from proximal femur, distal femur, proximal tibia, and lumbar spine in three periods respectively (8/12/16 weeks after the ovariectomy) to observe changes of different areas in bone morphology and density as well to identify areas sensitive to changes in bone mass and tissue structure, aiming to provide guidance for future experiments of postmenopausal osteoporosis research.

Animals

sixty SPF SD female rats of three months, non-pregnant, without offspring, weighing $(240 \pm 10)$ g, purchased from Shanghai Yukun Experimental Animals Co., Ltd.(Shanghai, China) under licence No. SCXK (Shanghai) 2016-0003; 10 clean SD female rats of three months, non-pregnant, without offspring, weighing $(240 \pm 10)$ g (Shanghai, China). The study was approved by the Animal Care and Use Committee of Fujian Academy of Traditional Chinese Medicine (protocol number: SYXK (Fujian) 2016-0005). 


\section{Main reagents and instruments}

Pentobarbital sodium (Sigma-Aldrich, St Louis, USA), paraformaldehyde (Sinopharm Chemical Reagent Co., Ltd., Shanghai, China), ponceau (Sinopharm Chemical Reagent Co., Ltd., Shanghai, China), fuchsin acid (Sinopharm Chemical Reagent Co., Ltd., Shanghai, China), Phosphomolybdic acid hydrate (Sinopharm Chemical Reagent Co., Ltd., Shanghai, China), fast green (Sinopharm Chemical Reagent Co., Ltd., Shanghai, China), Automatic tissue processor (Yaguang, ZT-14V2, Xiaogan, China), Embedding Center (Yaguang, YB-7LF, Xiaogan, China), Fully Automated Rotary Microtome (Leica, RM2255, Heidelberg, GER), Slide digital scanner (3D Histech, Pannoramic 250, Pannoramic MIDI, Budapest, HUN), Dual-energy X-ray scanner (Hologic, Discovery WS/N 89006, Boston, USA).

\section{Experimental design}

After being raised to 6 months, the SD rats were randomly divided into two groups: the sham operation group and operation group, each consisting of 30 rats. Bilateral ovariectomy was conducted to establish the postmenopausal osteoporosis model ${ }^{16}$. The rats were fed with water only and weighed one day before the operation. After anesthesia was done by injecting $2 \%$ pentobarbital sodium $(0.2 \mathrm{ml} / 100 \mathrm{~g})$ into the abdominal cavity, the rats were immobilized in the supine position and then shaved. A $1.5 \mathrm{~cm}$ incision was made on the midline of the abdomen. Then the abdomen was opened along the linea alba to find pink mulberry-shaped ovaries along the Y-shaped uterus. Ligation was done and ovaries were removed completely. After layer-by-layer suturing, 80,000 units penicillin was delivered through intramuscular injection for three days. The same methods were used in the sham operation group to remove the equal amount of adipose tissue near the ovaries. Samples were collected for three times in the 8th, 12th, and 16th week after the operation. The rest 10 female rats were raised to 24 months, without being intervened.

\section{Pathological observation}

The right tibiae, femur, and Lumbar spine 3-5 were collected and fixed in $4 \%$ paraformaldehyd at room temperature for 48 hours. Then the samples were decalcified with $10 \%$ EDTA ( $\mathrm{pH}$ 7.2-7.4), which was replaced every 3 days. After 6 weeks of decalcification at room temperature, when there was no resistance during knifing, the samples were washed with water, soaked with $70 \%$ ethyl alcohol for 1 hour, $80 \%$ ethyl for 1 hour, $90 \%$ ethyl for 1 hour, $95 \%$ ethyl for 1 hour, anhydrous ethanol I for 30 minutes, anhydrous ethanol II for 30 minutes, anhydrous ethanol III for 1 hour, xylene I for 15 minutes, xylene II for 25 minutes, paraffin I for 20 minutes, paraffin II for 20 minutes, and paraffin III for 40 minutes, and paraffin-embedded.

To ensure consistency, the slices were cut at the center of the samples, coronally in the tibia and femur and sagittally in the lumbar spine. The $4 \mu \mathrm{m}$ serial sections were heated in 60 degrees Celsius for 2 hours, dewaxed and put into water. Then they were fixed in Bouin's Fluid for 1 hour, washed with water until they became colorless, transferred to $10 \%$ ponceau staining solution for 10 minutes, separated colors with $1 \%$ phosphomolybdic acid hydrate, observed under the microscope, and fixed in $1 \%$ fast green staining solution for 1 minute. Then the slides were mounted using neutral balsam and scanned panoramically in the bright field to observe changes in bone morphology.

\section{Bone mineral denisty measurement}

First, the muscles and soft tissue on the proximal tibia and femur were removed as far as possible and the rest were wrapped in sa- line-wetted gauze for storage. Then dual-energy X-ray scanner was set to animal scanning mode. After the selection of ROI area, the bone density of the samples were read and the results printed out.

\section{Statistical analysis}

Results are represented as the mean \pm standard deviation and analyzed by using the SPSS 20.0 for Windows. Initial data followed normal distribution in the two groups was tested with Independent Samples T Test and non-normal data were verified with Non-parametric Testing. $P<0.05$ was considered statistically significant.

\section{Results}

\section{Masson staining}

After Masson staining, in the sham operation group it can be seen that under the epiphyseal line (marked in a black arrow), trabeculae are orderly, evenly distributed and closely spaced, interconnected to form a network, no obvious aggregation has been discovered in the medullary cavity. In contrast, samples in the operation group have sparse, disordered, unevenly distributed trabeculae with widened space and points of fracture, and a significantly increased amount of lipid droplets are observed in the medullary cavity. After the bilateral ovariectomy, the degree of trabecula loss increases with time, but in the same period the loss varies in different areas of the rats.

The proximal femur has a low level of bone loss: in the 8th and 12th week after the operation, trabecula loss is not significant and in the 16th week trabecula loss and a small amount of lipid droplets in the medullary cavity were found (Fig.1). Bone loss in the distal femur is significant: in the 8th week after the operation, an obviously loose and porous structure was observed in the samples and the degree increases over time; areas of trabecula loss spread from the center of the medullary cavity to the cortex of both sides, with an accumulation of lipid droplets (Fig.2). Proximal tibial bone loss is also significant, similar to the situation of the distal femur: areas of significant trabecula loss spread from the center of the medullary cavity to the cortex of the fibula side, with a large accumulation of lipid droplets (Fig.3). Lumbar spine bone loss is less significant: areas of trabecula loss spread from the center of the centrum to the disks of both sides, with a small quantity of lipid droplets (Fig.4). In the 16th week after the operation, for instance, bone loss is significant in the distal femur and proximal tibia and not significant in the proximal femur and lumbar spine.

\section{BMD}

In the 16th week after the operation bone loss is the most significant. If we look at both groups in this period, it can be found that compared with the sham operation group, bone density declines in all the four areas, and the differences have a statistical significance. The differences with the sham operation group, in descending order, are the proximal tibia, distal femur, proximal femur, and lumbar spine, which is basically consistent with the results from Masson staining (Table 1).

\section{4-month natural aging rats masson staining}

Sparse, fractured, disordered trabeculae can be seen in all the four areas. Trabecula loss is not severe in the epiphyseal line under the proximal femur and there is less lipid accumulation. Both trabecula loss and lipid accumulation are significant in the lumbar spine. Trabecula loss in the distal femur and proximal tibia is most severe and there is a significant lipid accumulation. Bone tissue change of natural aging rats is similar to that of ovariectomized rats in all the four areas (Fig.5). 
Sainan Chen et al.: Histomorphology and BMD in Ovariectomized Rats

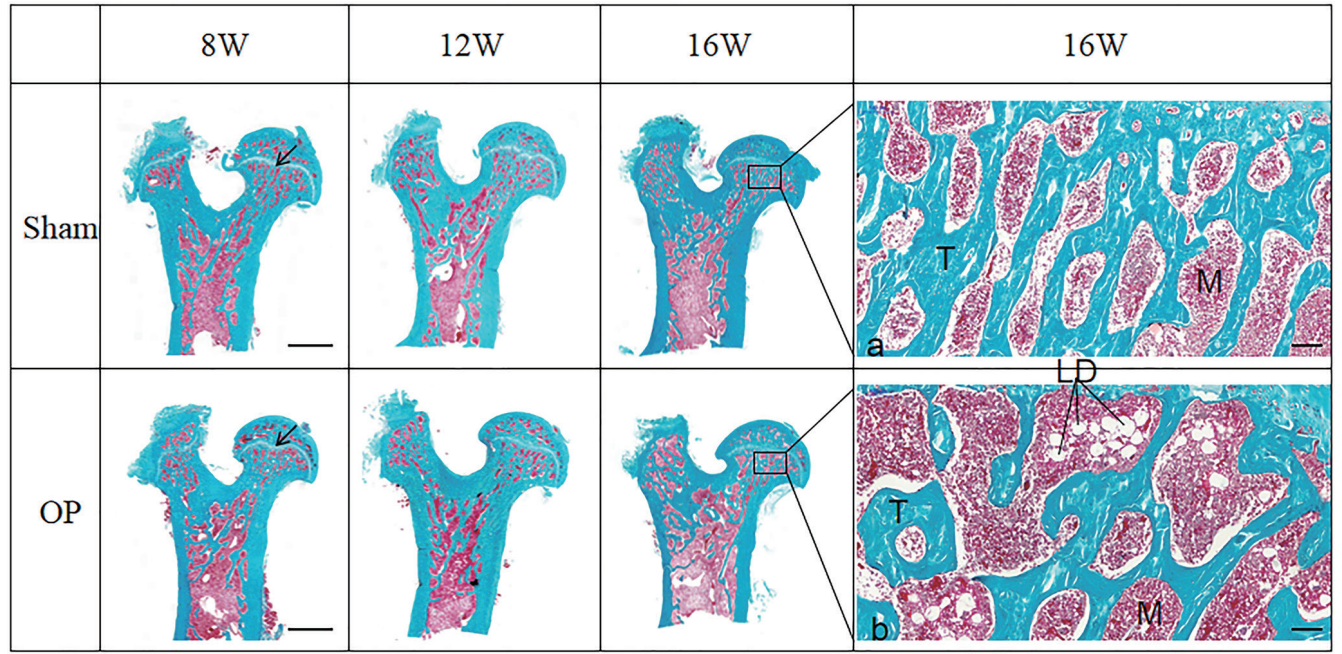

Figure 1. The coronal plane of proximal femoral. (Masson staining, Bar: 2,000 $\mu \mathrm{m}$, ab Bar: $100 \mu \mathrm{m}$ ) T:Trabecular bone, M:Marrow, LD: Lipid Droplet.

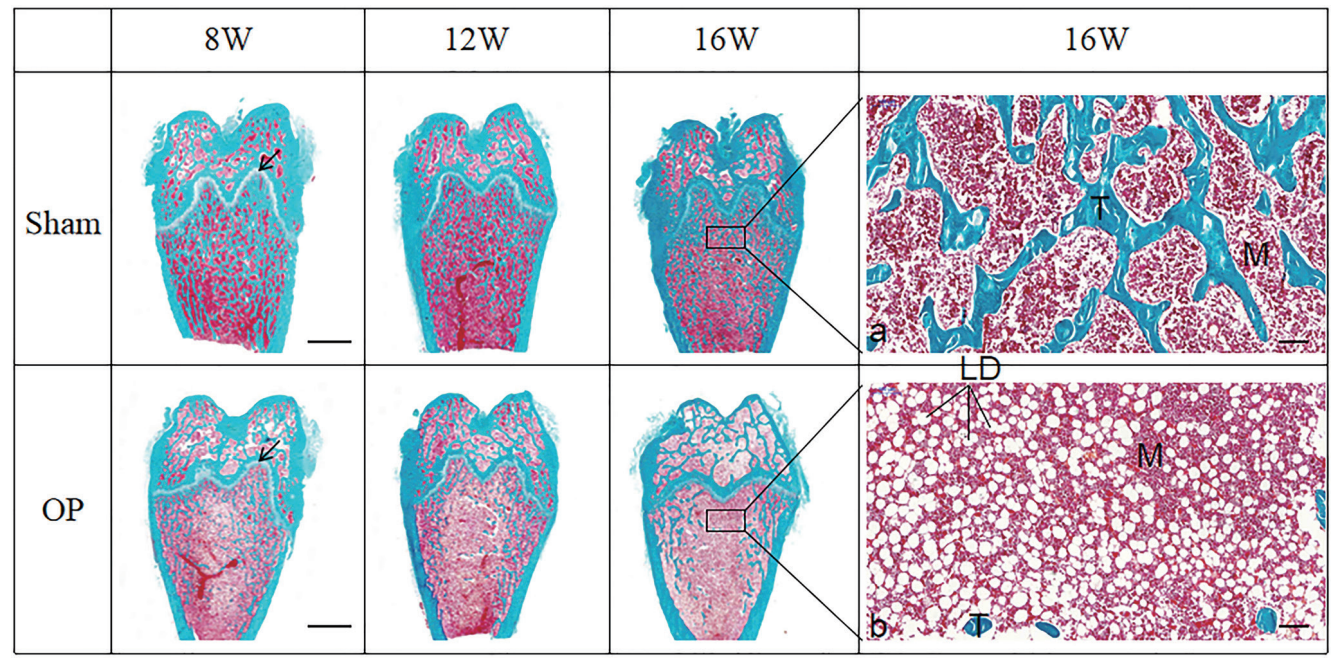

Figure 2. The coronal plane of distal femoral. (Masson staining, Bar: 2,000 $\mu \mathrm{m}$, ab Bar: $100 \mu \mathrm{m}$ )

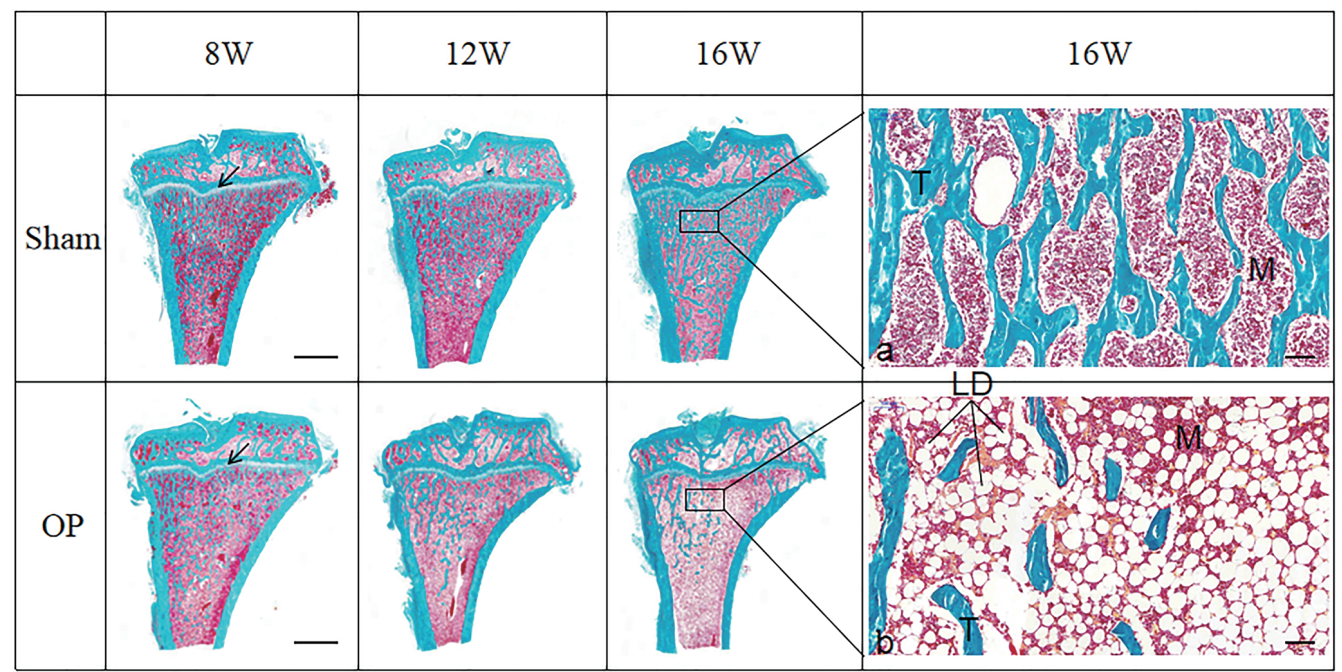

Figure 3. The coronal plane of proximal tibia. (Masson staining, Bar: 2,000 $\mu \mathrm{m}$, ab Bar: $100 \mu \mathrm{m}$ ) 


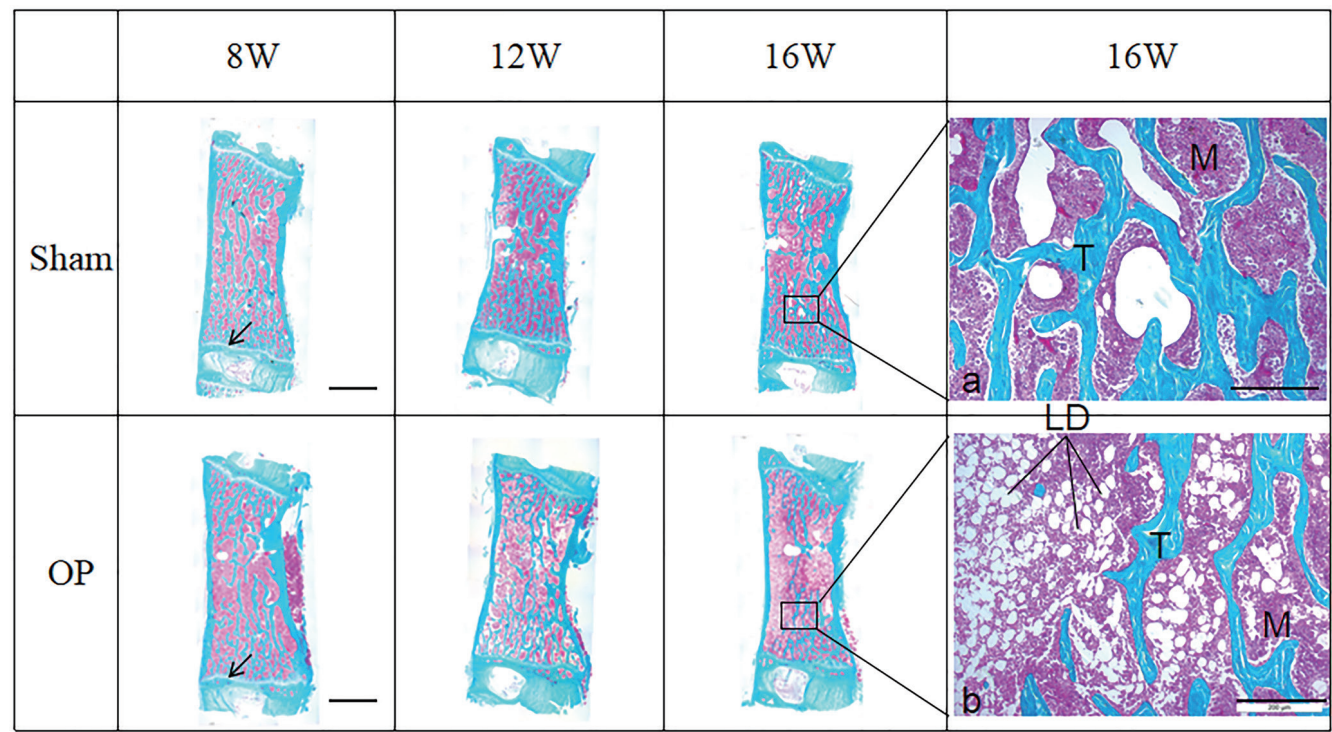

Figure 4. The sagittal plane of lumbar spine. (Masson staining, Bar: 2,000 $\mu \mathrm{m}$, ab Bar: $200 \mu \mathrm{m}$ )

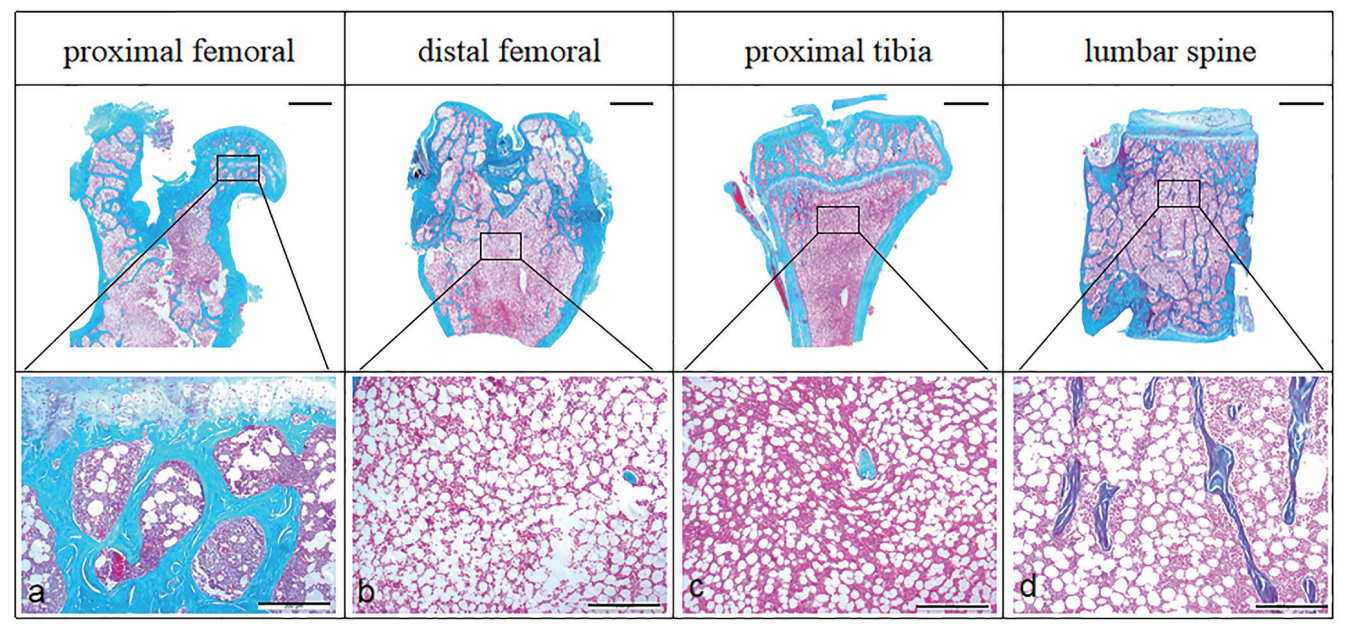

Figure 5. 24-month Natural Aging Rats. (Masson staining, Bar: 2,000 $\mu \mathrm{m}$, abcd Bar: $200 \mu \mathrm{m}$ )

Table 1. BMD in the 16 th week after operation $\left(\mathrm{g} / \mathrm{cm}^{2}\right)$

\begin{tabular}{ccccc}
\hline & Proximal femur & Distal femur & Proximal tibia & Lumbar spine \\
\hline Sham-16W & $0.30975 \pm 0.0109$ & $0.4398 \pm 0.0214$ & $0.4168 \pm 0.0212$ & $0.2001 \pm 0.0071$ \\
OP-16W & $0.2869 \pm 0.01^{*}$ & $0.3849 \pm 0.0214^{*}$ & $0.3321 \pm 0.0101^{*}$ & $0.1801 \pm 0.0078^{*}$ \\
Difference & $0.0228 \pm 0.0164$ & $0.0549 \pm 0.0329$ & $0.0847 \pm 0.0259$ & $0.0196 \pm 0.0067$ \\
Statistics & $\mathrm{T}=4.366 P=0.001$ & $\mathrm{~W}=36 P=0.000$ & $\mathrm{~W}=36 P=0.000$ & $\mathrm{~T}=5.607 P=0.000$ \\
\hline
\end{tabular}

*: $P<0.01$, compared to the sham operation group 


\section{Discussion}

PMOP is a high bone transformation osteoporosis. It is a systemic bone disease that generally develops in women after menopause when the ovaries decline in function and the amount of estrogen decreases, causing overactive bone resorption and imbalanced bone remodeling and further leading to reduced bone mass, destroyed bone microstructure, increased bone fragility, and a higher risk of fracture ${ }^{17)}$. The bone remodeling threshold is 200 microstrains. Due to the sharp decline in estrogen level after menopause, the threshold increases by around $30 \%$, activation frequency significantly rises, bone resorption exceeds bone formation, newly formed osteon shows negative balance, and total bone mass reduces ${ }^{18,19)}$. Bilateral ovariectomy in female rats is one of the most commonly used animal model during research, which can satisfactorily simulate the pathological state of PMOP and is widely employed in research on the efficacy and mechanisms of anti-osteoporosis drugs ${ }^{20,21)}$.

DXA is the gold standard diagnostic procedure used to detect primary osteoporosis. At axial bones and/or $1 / 3$ of nondominant distal radius, when bone density $\mathrm{T}$ score is above $-1 \mathrm{SD}$, bone mass is considered to be at normal level, while $-2.5 \mathrm{SD} \leq \mathrm{T}<-1 \mathrm{SD}$ suggests low bone mass, and $\mathrm{T}<-2.5$ is classified into osteoporosis. $\mathrm{T}<-2.5 \mathrm{SD}$ accompanied by fracture of one site or more suggests serious osteoporosis ${ }^{22-24)}$. Yet BMD change cannot fully reflect changes in bone strength. Quantitative computed tomography (QCT) allows us to obtain three-dimensional data on the density and morphological structure of cancellous bones and cortical bones to realize three-dimensional structure analysis. By detecting the density of cancellous bones, QCT can reflect more accurately the metabolism change of osteoporosis than DXA do ${ }^{25,26)}$. In Expert Consensus on the Diagnosis of Osteoporosis, Zhang Zhihai et al. proposed for the first time in China using "lumbar spine BMD below $80 \mathrm{mg} / \mathrm{cm}^{3}$ by QCT"recommended by International Society for Clinical Densitometry and American College of Radiology — as a diagnostic criteria ${ }^{27)}$. The low resolution of $0.5 \mathrm{~mm}$ makes QCT unable to evaluate the conditions of trabeculae. Yet the high resolution quantitative CT $(80 \mu \mathrm{m})^{28)}$ can only be used in peripheral bones, due to the partial volume effect. With a high resolution between $5 \mu \mathrm{m}$ and $35 \mu \mathrm{m}$, micro-CT can realize the 3D structure remodeling and analysis of bone tissue and trabeculae ${ }^{29,30)}$, but the high cost prohibits its widespread use. At present there is no approach to directly measure bone quality and in most cases BMD measurement is used as a surrogate. Hence basic research on osteoporosis mostly performs a comprehensive analysis of BMD and morphology.

Bone morphology can show clearly the amount, thickness, spacing, and structure of trabeculae, the amount and distribution of osteoblasts and osteoclasts, as well as changes in the medullary cavity and cellular contents, which, when combined with tetracyclin and calcein, can dynamically analyze the mineral apposition rate and bone formation rate $^{31,32)}$ and accurately reflect the quality of bone structures. Nonetheless, it has two disadvantages. First, it observes a rather limited range, for the higher magnification of the objective lens is, the smaller the bone tissue areas can be presented under the lens, which easily leads to errors in analysis. Thus low power objective lens should be used first to observe samples in large areas, or slide scanning be employed to produce panoramic digital sections that can support observation from $1 \times$ to $400 \times$ to reduce information loss of the sections and avoid objective deviation caused by the analyzing different areas. Second, as the sections to be observed are two-dimensional, the sections of different samples are required to be cut at similar sites so as to enhance the reliability of comparative analysis and to avoid objective deviation caused by analyzing varied areas. Taken together, it is recommended that low-power whole slide imaging of similar sites be used for osteoporosis sections, in order to more reliably reflect the structural changes of bone tissue, and measurement results from DXA and QCT be considered together for comprehensive analysis.

In physiological state, there are differences in bone tissue between different regions of skeleton, such as trabecular bone structure, bone mineral density and bone tissue mechanics. The loss of bone mass in osteoporosis resulted in the redistribution of lost bone tissue, which aggravated the heterogeneity of bone tissue in different regions. The spine, as the axial bone in upright-walking humans, bears most of the weight especially the lumbar spine. Yet for quadrupedal-walking rodents, the lumbar spine mainly receives stress of muscle contraction, while the femur and tibia receives extra stress from load-bearing. Moreover, the cortical bone of rats lacks Haversian system, the activity of bone remodeling is low, and the change of bone mass is not significant. The sensitive area of bone mass change is cancellous bone. Therefore, the abundant area of cancellous bone mass in rats was observed in this study. The experiment in this study selects the 8 th, 12th, and 16th week after the bilateral ovariectomy to compare the changes in bone morphology and density at the proximal femur, distal femur, proximal tibia, and lumbar spine respectively, aiming to find areas in postmenopausal osteoporotic rats that are sensitive to bone mass changes. The results show that the degree of trabecula loss in the same area increases over time after the ovariectomy and in the 16th week bone loss is the most significant. In the same period the loss varies in different parts of the rats - the differences in bone density, in descending order, are the proximal tibia, distal femur, proximal femur, and lumbar spine. Results from Masson staining are consistent with that from bone density measurement. Moreover, a positive correlation has been found between the degree of trabecula loss and lipid accumulation. The bone mass change and lipid accumulation in the cavity of 24-month naturally aging rats are consistent with rats after bilateral ovariectomy. The laboratory findings show that unlike humans, areas in rats that are sensitive to osteoporosis bone mass changes are major weight-bearing bones: proximal tibia and distal femur.

Different degrees of stress received by different areas of the same bone determines that trabeculae are not evenly distributed, which is more typical in bones bearing a major physiological load. The laboratory findings show that at the proximal tibia, areas of significant trabecula loss spread from the center of the medullary cavity to the cortex of the fibula side and at the distal femur bone loss is the most significant in the center of the medullary. Trabecula loss starts from bones that function the least, namely, the non-weight-bearing areas, and the weight-bearing areas degenerate slowly and lose the least amount of trabeculae. In the same bone, non-weight-bearing areas are more sensitive to bone mass change than weight-bearing areas. Bones form the optimal structure to ensure the most basic weight-bearing and to delay or prevent fractures. In this experiment, trabecula redistribution in osteoporosis is in agreement with Wolff's law ${ }^{33,34)}$, which states that bone's form follows its function. Every change in the form and function of bone, or of their function alone, is followed by certain definite changes in their internal architecture and equally definite secondary alteration in their external confor mation, in accordance with mathematical laws ${ }^{33,34)}$. More specifically, bone adjusts its geometrical shapes and internal architecture to achieve the maximum bearing capacity with the optimal shapes and structure and minimum mass ${ }^{35-39}$. When the loading increases, it changes accordingly to adapt to the situation with with the optimal shapes and structure. Through continuous remodeling and optimization, bone tissue maintains the dynamic balance structurally and functionally ${ }^{40)}$.

Bone marrow mesenchymal stem cells (BMSCs) are derived from 
mesoderm and capable of self-renewal and multidirectional differentiation. It can differentiate into chondrocyte, osteocyte, adipocyte, hepatocyte and other cells under different induction conditions. The number and activity of BMSCs play a vital role in promoting bone formation, maintaining bone mass and enhancing bone mineral density ${ }^{41)}$. Normally, BMSCs have the same potential to differentiate into osteoblasts and adipocytes. However, in some diseases, the balance will be broken, and the differentiation of BMSCs into one aspect of osteogenesis or adduction will be enhanced, while differentiation into the other direction will be weakened ${ }^{42)}$. The results of this study showed that BMSCs were imbalanced in osteogenic or adipogenic differentiation in PMOP, the ability of osteogenic differentiation was diminished, and the ability of adipogenic differentiation was enhanced, which manifested as bone loss and accumulation of adipocytes in medullary cavity, and the number of adipocytes increased with the prolongation of the course of disease. Therefore, promoting the differentiation of BMSCs into osteoblasts is of great significance for the treatment of PMOP.

Our results showed that areas sensitive to bone mass change in ovariectomized rats are load-bearing bones - the proximal tibia and distal femur. Trabecula loss is most significant in non-weight-bearing areas and there is a positive correlation between the degree of trabecula loss and lipid accumulation in the medullary cavity, making such areas optimal for osteoporosis research on postmenopausal rats.

\section{Acknowledgments}

This work was supported by the Fundamental Research Project for Common Wealth Scientific Institutes in Fujian Province (Nos.2017R1035-4), the subject of Health and Family Planning Youth Scientific Research in Fujian Province (Nos.2017-1-79), the subject of traditional Chinese Medicine Research of Fujian Health and Family Planning Commission (Nos.2017FJZYLC102), the Fujian Provincial Natural Science Foundation Projects (Nos.2017J01332).

\section{Conflict of Interest}

The authors declare that no conflict of interest exists.

\section{References}

1. NIH consensus development panel on osteoporosis prevention, diagnosis, and therapy, osteoporosis prevention, diagnosis, and therapy. JAMA 285: 785-795, 2001

2. Lorentzon M and Cummings SR. Osteoporosis: the evolution of a diagnosis. J Intern Med 277: 650-661, 2015

3. Eastell R, O'Neill TW, Hofbauer LC, Langdahl B, Reid IR, Gold DT and Cummings SR. Postmenopausal osteoporosis. Nat Rev Dis Primers 2: 16069, 2016

4. Rachner TD, Khosla S and Hofbauer LC. Osteoporosis: now and the future. Lancet 377: 1276-1287, 2011

5. Chen $\mathrm{P}, \mathrm{Li} \mathrm{Z}$ and $\mathrm{Hu}$ Y. Prevalence of osteoporosis in China: a meta-analysis and systematic review. BMC Public Health 16: 1039, 2016

6. Cauley JA. Osteoporosis: fracture epidemiology update 2016. Curr Opin Rheumatol 29: 150-156, 2017

7. Lin X, Xiong D, Peng YQ, Sheng ZF, Wu XY, Wu XP, Wu F, Yuan LQ and Liao EY. Epidemiology and management of osteoporosis in the People's Republic of China: current perspectives. Clin Interv Aging 10: 1017-1033, 2015

8. Solomon DH, Patrick AR, Schousboe J and Losina E. The potential economic benefits of improved postfracture care: a cost-effectiveness analysis of a fracture liaison service in the US health-care sys- tem. J Bone Miner Res 29: 1667-1674, 2014

9. Lötters FJ, van den Bergh JP, de Vries F and Rutten-van Mölken MP. Current and future incidence and costs of osteoporosis-related fractures in the Netherlands: Combining claims data with BMD measurements. Calcif Tissue Int 98: 235-243, 2016

10. Amugongo SK, Yao W, Jia J, Dai W, Lay YA, Jiang L, Harvey D, Zimmermann EA, Schaible E, Dave N, Ritchie RO, Kimmel DB and Lane NE. Effect of sequential treatments with alendronate, parathyroid hormone (1-34) and raloxifene on cortical bone mass and strength in ovariectomized rats. Bone 67: 257-268, 2014

11. Saul D, Gleitz S, Nguyen HH, Kosinsky RL, Sehmisch S, Hoffmann DB, Wassmann M, Menger B and Komrakova M. Effect of the lipoxygenase-inhibitors baicalein and zileuton on the vertebra in ovariectomized rats. Bone 101: 134-144, 2017

12. Seif AA. Nigella Sativa reverses osteoporosis in ovariectomized rats. BMC Complement Altern Med 14: 22, 2014

13. Sun X, Li F, Ma X, Ma J, Zhao B, Zhang Y, Li Y, Lv J and Meng X. The effects of combined treatment with naringin and treadmill exercise on osteoporosis in ovariectomized rats. Sci Rep 5: 13009, 2015

14. Mathavan N, Turunen MJ, Tägil M and Isaksson H. Characterising bone material composition and structure in the ovariectomized (OVX) rat model of osteoporosis. Calcif Tissue Int 97: 134-144, 2015

15. Paolillo FR, Romano RA, de Matos L, Martin AA, Guimarães FEG, de Castro Neto JC and Bagnato VS. Short-term and long-term effects of osteoporosis on incisor teeth and femoral bones evaluated by Raman spectroscopy and energy dispersive X-ray analysis in ovariectomized rats. J Bone Miner Metab 2018 Jan 17. doi: 10.1007/s00774-018-0903-6.

16. Komori T. Animal models for osteoporosis. Eur J Pharmacol 759: 287-294, 2015

17. Management of osteoporosis in postmenopausal women: 2010 position statement of The North American Menopause Society. Menopause 17: 25-54, 2010

18. Schiessl H, Frost HM and Jee WS. Estrogen and bone-muscle strength and mass relationships. Bone 22: 1-6, 1998

19. Ferretti JL, Schiessl H and Frost HM. On new opportunities for absorptiometry. J Clin Densitom 1: 41-53, 1998

20. Xu JH, Yao M, Ye J, Wang GD, Wang J, Cui XJ and Mo W. Bone mass improved effect of icariin for postmenopausal osteoporosis in ovariectomy-induced rats: a meta-analysis and systematic review. Menopause 23: 1152-1157, 2016

21. Xing LZ, Ni HJ and Wang YL. Quercitrin attenuates osteoporosis in ovariectomized rats by regulating mitogen-activated protein kinase (MAPK) signaling pathways. Biomed Pharmacother 89: 1136-1141, 2017

22. Assessment of fracture risk and its application to screening for postmenopausal osteoporosis. Report of a WHO Study Group. World Health Organ Tech Rep Ser 843: 1-129, 1994

23. Kanis JA, Melton LJ 3rd, Christiansen C, Johnston CC and Khaltaev N. The diagnosis of osteoporosis. J Bone Miner Res 9: 11371141, 1994

24. Kanis JA, McCloskey EV, Johansson H, Oden A, Melton LJ 3rd and Khaltaev N. A reference standard for the description of osteoporosis. Bone 42: 467-475, 2008

25. Eriksen EF, Keaveny TM, Gallagher ER and Krege JH. Literature review: The effects of teriparatide therapy at the hip in patients with osteoporosis. Bone 67: 246-256, 2014

26. Engelke K, Lang T, Khosla S, Qin L, Zysset P, Leslie WD, Shep- 
herd JA and Schousboe JT. Clinical use of quantitative computed tomography (QCT) of the hip in the management of osteoporosis in adults: the 2015 ISCD official positions-part I. J Clin Densitom 18: 338-358, 2015

27. ZHANG Zhihai, LIU Zhonghou and LI Na. The osteoporosis committee of China gerontological society, expert consensus on the diagnosis of osteoporosis in Chinese population. Chin J Osteopor 20: 1007-1010, 2014

28. Cheung AM, Adachi JD, Hanley DA, Kendler DL, Davison KS, Josse R, Brown JP, Ste-Marie LG, Kremer R, Erlandson MC, Dian L, Burghardt AJ and Boyd SK. High-resolution peripheral quantitative computed tomography for the assessment of bone strength and structure: a review by the Canadian Bone Strength Working Group. Curr Osteoporos Rep 11: 136-146, 2013

29. Effendy NM, Khamis MF and Shuid AN. Micro-CT assessments of potential anti-osteoporotic agents. Curr Drug Targets 14: 15421551,2013

30. Wu Y, Adeeb S and Doschak MR. Using micro-CT derived bone microarchitecture to analyze bone stiffness - a case study on osteoporosis rat bone. Front Endocrinol (Lausanne). 6: 80, 2015

31. Takakura A, Lee JW, Hirano K, Isogai Y, Ishizuya T, Takao-Kawabata R and Iimura T. Administration frequency as well as dosage of PTH are associated with development of cortical porosity in ovariectomized rats. Bone Res 5: 17002, 2017

32. Zheng LZ, Wang XL, Cao HJ, Chen SH, Huang L and Qin L. Src siRNA prevents corticosteroid-associated osteoporosis in a rabbit model. Bone 83: 190-196, 2016
33. Wolff J. Das Gesetz der transformation der Knochen. Berlin: Hirschwald.1892

34. Wolf JH. Julis Wolff and his "law of bone remodeling". Orthopade 24: 378-386, 1995

35. Frost HM. The laws of bone structure. Springfield, IL: Thomas. 1964

36. Frost HM. Bone "mass" and the "mechanostat": a proposal. Anat Rec 219: 1-9, 1987

37. Frost HM. From Wolff's law to the utah paradigm: insights about bone physiology and its clinical applications. Anat Rec 262: 398419, 2001

38. Lanyon LE. The success and failure of the adaptive response to functional load-bearing in averting bone fracture. Bone 13: 17-21, 1992

39. Barak MM, Lieberman DE and Hublin JJ. A Wolff in sheep's clothing: trabecular bone adaptation in response to changes in joint loading orientation. Bone 49: 1141-1151, 2011

40. Roux W. Gesammelte abhandlungen. Vol. 1. Leipzig: Engelmann. 1895

41. Vial IN and Peter Rubin J. Commentary on: isolation and differentiation potential of human mesenchymal stem cells from adipose tissue harvested by water jet-assisted liposuction. Aesthet Surg J 35:1040-1041, 2015

42. Palermo A, Tuccinardi D, Defeudis G, Watanabe M, D’Onofrio L, Lauria Pantano A, Napoli N, Pozzilli P and Manfrini S. BMI and BMD: The potential interplay between obesity and bone fragility. Int J Environ Res Public Health 13: 544, 2016 
J.Hard Tissue Biology Vol. 28(2): 199-206, 2019 